\title{
Throughput Improvement by Adjusting RTS Transmission Range for W-LAN Ad Hoc Network
}

\author{
Akihisa Matoba \\ Graduate School of Informatics, \\ Tokyo University of \\ Information Sciences, \\ 4-1 Onaridai, Wakaba-ku, \\ Chiba, 265-8501 Japan
}

\author{
Masaki Hanada \\ Department of Informatics, \\ Tokyo University of \\ Information Sciences, \\ 4-1 Onaridai, Wakaba-ku, \\ Chiba, 265-8501 Japan
}

\author{
Moo Wan Kim \\ Department of Informatics, \\ Tokyo University of \\ Information Sciences, \\ 4-1 Onaridai, Wakaba-ku, \\ Chiba, 265-8501 Japan
}

\begin{abstract}
The W-LAN Ad Hoc network tends to cause problems called "Hidden Node" and "Exposed Node". RTS/CTS mechanism has been introduced to mitigate Hidden Node and most of existing researches assume that RTS and CTS are sent at the same transmission range. This paper describes a new method to improve the network throughput by adjusting the RTS transmission range. The simulation result showed that the proposed method achieved higher throughput in some degree.
\end{abstract}

\section{INTRODUCTION}

T HE W-LAN Ad Hoc network tends to cause problems called "Hidden Node" and "Exposed Node" [1]. Fig.1 shows an example of Hidden Node and Exposed Node. In Fig.1, transmission range and receive range are assumed to be equal. Assuming a sender node and a receiver node, a Hidden Node is located near to the receiver node and can hear the transmission from the receiver node while it cannot hear the transmission from the sender node as it is far enough from the sender node. The Hidden Node and the sender node can send a frame respectively at the same moment and can cause a collision at the receiver node. An Exposed Node is located near the sender node and its transmission reaches to the sender node but cannot reach to the receiver node as it is far enough from the receiver node. The Exposed Node doesn't cause a collision at the receiver node when it sends a frame at the same moment when the sender node sends a frame. But due to carrier sense mechanism of IEEE802.11, the Exposed Node detects a transmission of the sender node and has it suspend the transmission. This may cause unnecessary transmission suspensions and degrade network performance [2].

RTS/CTS mechanism as shown in Fig. 2 has been introduced to mitigate Hidden Node since the first version of IEEE802.11 [3].

First a sender node does carrier sense. If channel is idle, it further waits DIFS (DCF Inter Frame Space) period and random back off period. Then it sends RTS (Request To Send) and any nodes which hear the RTS reserves air time during

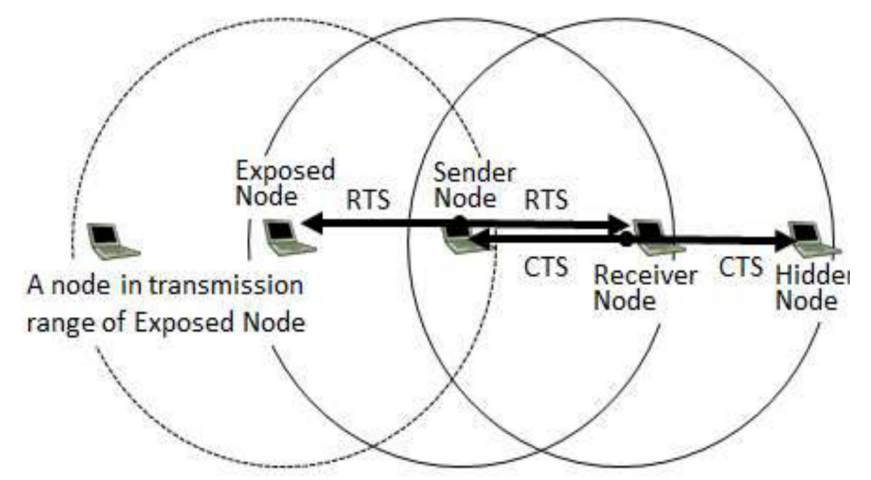

Fig.1 Example of Hidden Node and Exposed Node

NAV (Network Allocation Vector) period. The receiver node receives RTS and sends back CTS (Clear To Send) after SIFS (Short Inter frame Space) period.

CTS also have NAV and suspend transmission of nodes which hear the CTS until the receiver node sends ACK. Then the sender node sends a data frame after it receives CTS and the receiver node sends ACK after it receives the data frame. In this mechanism, Hidden Nodes around the receiver node can suspend transmissions by the CTS and the receiver node can avoid collisions to receive the data frame. But this mechanism creates Exposed Nodes around the sender node as they hear the RTS and suspend their transmission.

In this paper a new RTS/CTS method to reduce the number of Exposed Nodes has been proposed. This method assigns different transmission range to RTS and CTS respectively to reduce the number of Exposed Nodes. Simulation results shows that the proposed method improves the entire network throughput compared to the standard RTS/CTS mechanism, and also has effect to equalize variation of throughput among each node. 


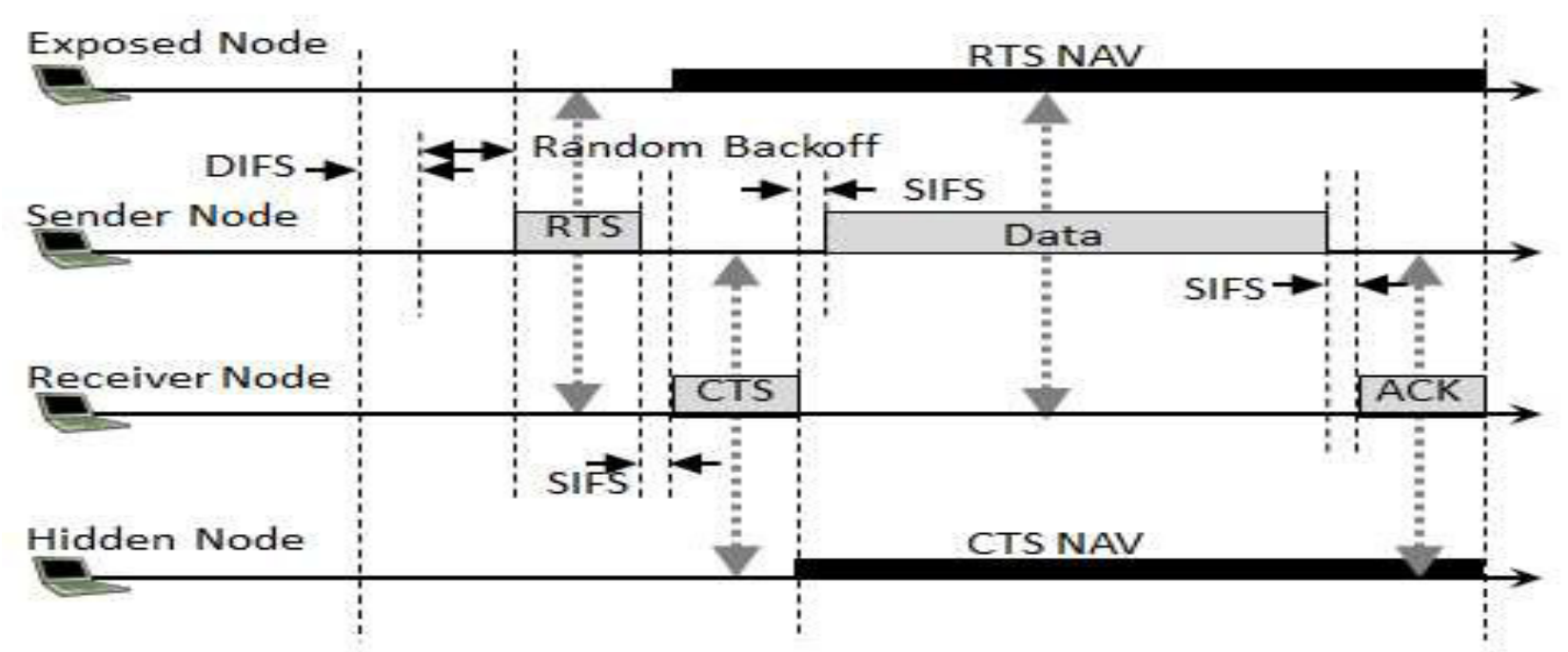

Fig. 2 Standard RTS/CTS Mechanism

\section{PROPOSED METHOD}

\section{A. Related Work}

Various researches have been conducted to mitigate Exposed Node and Hidden Node [4]. The most of existing researches assume that RTS, CTS and Data frame are sent at the same data transmission rate and have the same radio coverage. This was true with the first version of IEEE802.11, but now Data frame is sent at multi rates (54Mbps with $11 \mathrm{a} / \mathrm{g}$ at maximum) while control frames such as RTS/CTS remain the lowest basic rate (1Mbps with $11 \mathrm{~g}, 6 \mathrm{Mbps}$ with 11a). There are some researches to consider multi transmission rate, but no researches assume different transmission rate to RTS and CTS.

\section{B. Basic Idea}

In our research we intentionally allocate different transmission rate to RTS and CTS in order to proactively control the radio coverage and mitigate effect of Exposed Node. We have redefined the objective of RTS only to provoke CTS. RTS needs to reach to receiver node but it doesn't need to reach to any other nodes. So the RTS should be sent at the maximum data rate as data frame. This strategy introduces risk to lose CTS and ACK at the sender node by collisions from surrounding nodes (Exposed Nodes). But we assume this risk is minimal as CTS and ACK have short length in comparison to data frame.

Fig. 3 shows the basic idea of our proposal. Here we define Sender Node as S, Receive Node as R, Hidden Node as $\mathrm{H}$ and Exposed nodes as $\mathrm{Ei}, \mathrm{Nj}$. Radius of $\mathrm{RTS}$ range and CTS range by standard method are defined as Rrts and Rcts. Radius of proposed higher transmission rate of RTS (i.e. RTS') is defined R'rts. As shown in Fig.3, higher transmission rate of RTS makes the RTS coverage range smaller than CTS and reduces Exposed Node. In order to avoid collision of data frame at the receiver node, CTS should be sent at lowest data rate to be heard by Hidden Node as many as possible. In case if RTS range is completely included in CTS range, there is no Exposed Node. The shaded area of Fig.3 contains the eliminated exposed nodes (i.e. $\mathrm{Nj}$ ) by proposal.

The following steps show the procedure of the proposal;

Step1: S sends RTS' to $\mathrm{R}$ with possible highest transmission rate. This is to minimize the RTS' coverage range and to reduce the number of Ei.

Step2: R receives RTS' and sends back CTS with basic transmission rate. This is to ensure all potential hidden nodes to receive CTS and to suspend their transmission.

Step3: $\mathrm{S}$ receives CTS and sends data frame to $\mathrm{R}$ with maximum transmission rate. If the RTS' range is completely included in the CTS range, there is no exposed node.

Step4: $\mathrm{R}$ receives data frame and sends back ACK with highest transmission rate.

By the way above discussion, to reduce radio coverage is not applicable to CCA (Clear Channel Assessment). Any frames have PLCP preamble and header with $1 \mathrm{Mbps}$, and the payload portion is in higher date rate (e.g. 54Mbps). The effect of CCA may need to be investigated further.

\section{SimULATION}

\section{A. Simulation Condition}

We have assumed Wireless LAN standard of $5 \mathrm{GHz}$ band, IEEE802.11a. The system parameter for the simulation is shown in Table 1. 


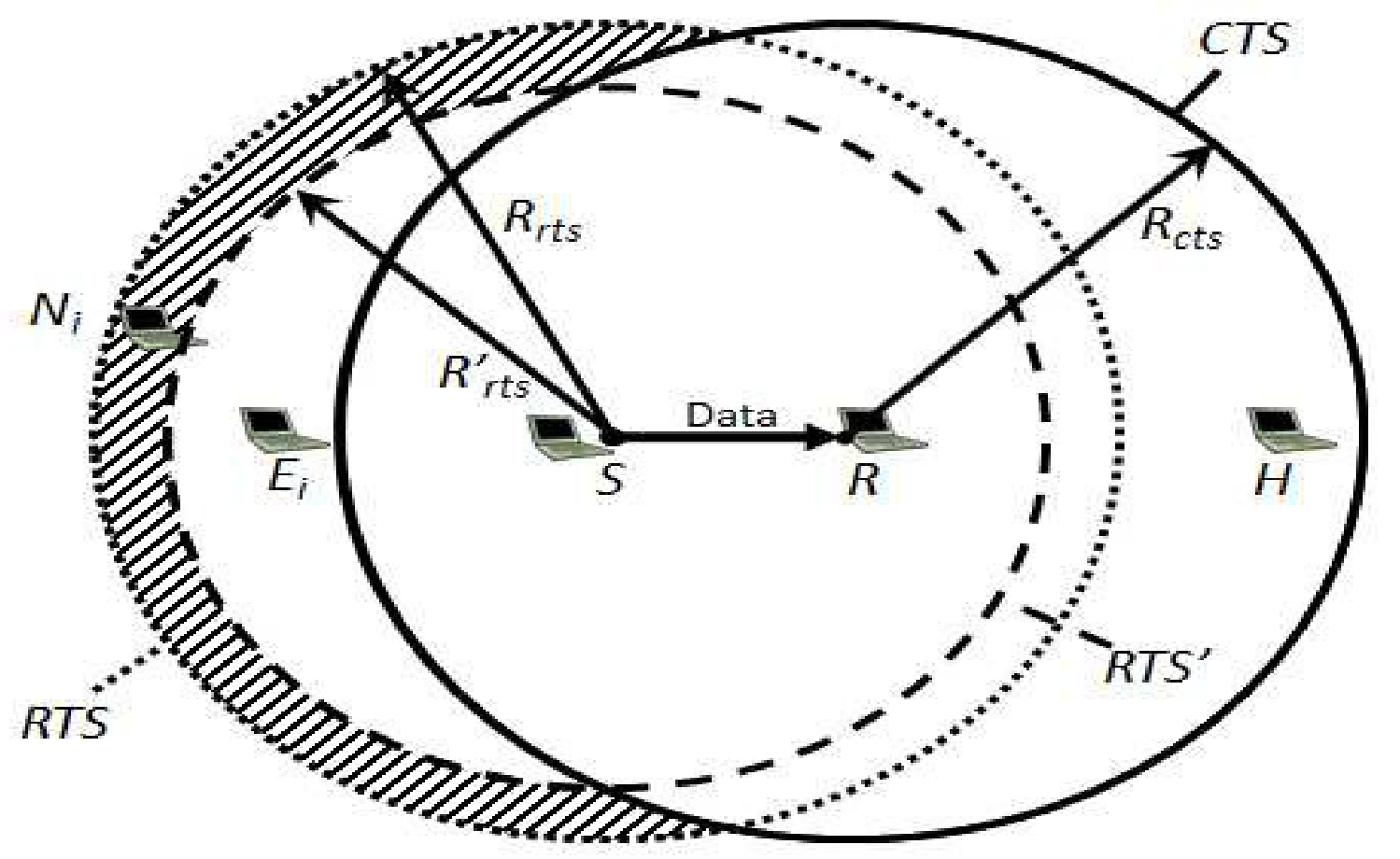

Fig. 3 Basic Idea of Proposal

In IEEE802.11a, 8 transmission rates are defined as $6 \mathrm{Mbps}$, 9Mbps, 12Mbps, 18Mbps, 24Mbps, 36Mbps, $48 \mathrm{Mbps}$ and $54 \mathrm{Mbps}$. For simplicity, in this simulation, RTS is sent at $18 \mathrm{Mbps}$ and CTS is sent at the minimum basic rate as $6 \mathrm{Mbps}$. Data and ACK are sent at the same as RTS (i.e. 18Mbps).

As the simulated network topology all nodes are located in a grid with $70 \mathrm{~m}$ interval. Seven cases are assumed for grid size from $3 \times 3$ with 9 nodes to $15 \times 15$ with 255 nodes. Nodes can be randomly distributed, but in practical deployment distribution of nodes is often governed by artificial objects such as walls, furniture, partitions and structures of building and it follows geometric arrangement. So we assumed the grid distribution as the initial research stage.

The $5 \times 5$ grid of nodes is shown in Fig.4. The node 13 is the sender node and receiver node is selected among node 8 , 12, 14 and 18 at random. In Fig.4, node 14 is selected as the receiver node.

TABLE 1.

SySTEM PARAMETERS FOR SimUlation

\begin{tabular}{|c|c|c|c|c|}
\hline \multirow[t]{5}{*}{ Frame } & Type & Transmission Rate & \multicolumn{2}{|l|}{ Range } \\
\hline & RTS & $18 \mathrm{Mbps}$ & $88 \mathrm{~m}$ & 1 hop (70m) \\
\hline & CTS & 6Mbps & $140 m$ & 2 hops (140m) \\
\hline & Data & $18 \mathrm{Mbps}$ & $88 \mathrm{~m}$ & 1 hop $(70 m)$ \\
\hline & ACK & $18 \mathrm{Mbps}$ & $88 \mathrm{~m}$ & 1 hop (70m) \\
\hline Load & \multicolumn{4}{|c|}{ 3Mbps per node with exponential distribution } \\
\hline Data Size & \multicolumn{4}{|c|}{1,000 bytes } \\
\hline Distance & \multicolumn{4}{|c|}{ Nodes are located at $70 \mathrm{~m}$ interval in a grid. } \\
\hline Other & \multicolumn{4}{|c|}{$\begin{array}{l}\text { DIFS }=34 \mu \mathrm{s}, \quad \text { SIFS }=16 \mu \mathrm{s} \text { and Slot time }=9 \mu \mathrm{s} \text {. Other parameters } \\
\text { follow } 802.11 \mathrm{a} \text { standard. }\end{array}$} \\
\hline
\end{tabular}




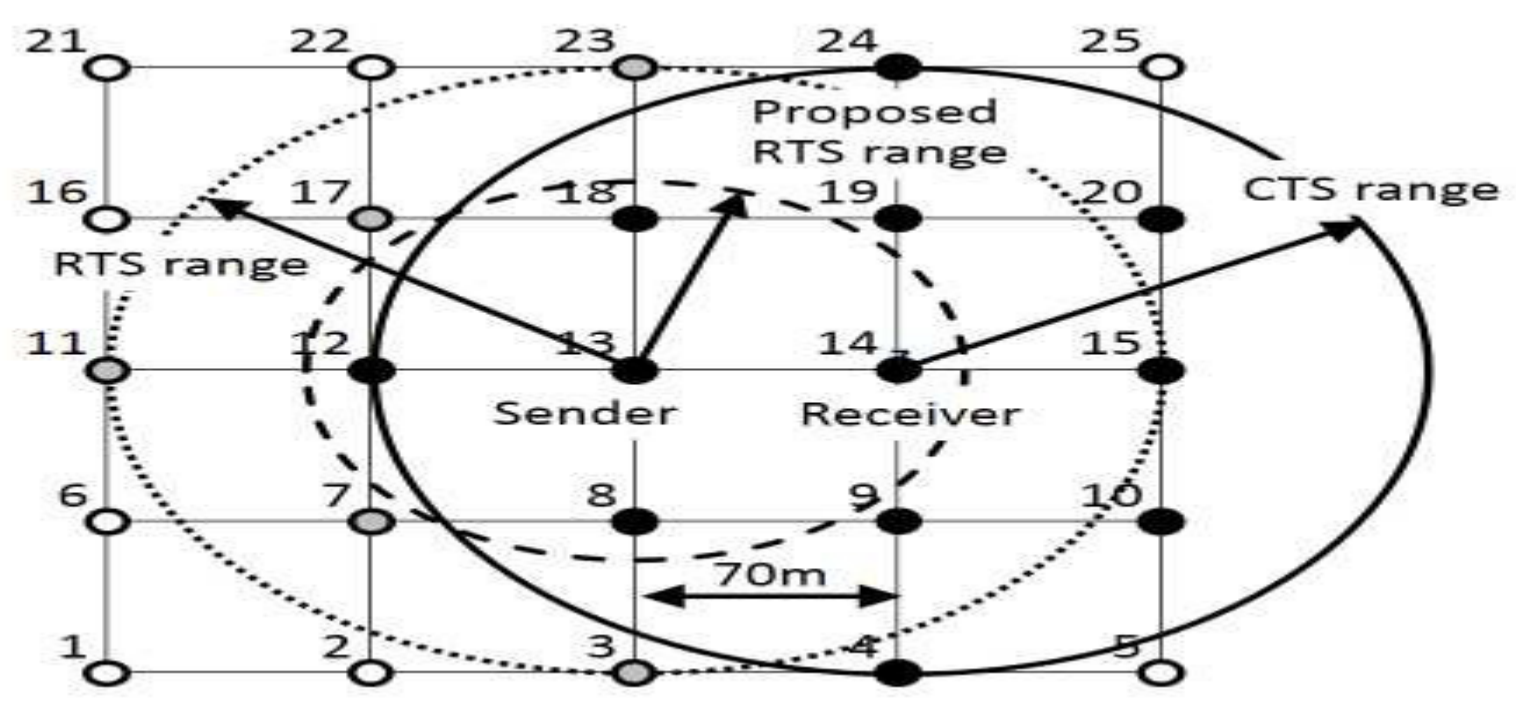

Fig. 425 Nodes (5 x 5) Example

An RTS with standard method reaches up to a node at two hop distance and totally 12 nodes excluding sender node are in transmission range. An RTS with proposed method reaches only to nodes at one hop distance and totally 4 nodes are in transmission range. As the CTS transmission range is two hops, RTS range of the proposed method is completely included in CTS range.

In Fig.4, black nodes are in the CTS transmission range and white nodes have no influences with the transmission from note 13 to node 14. Gray nodes would be Exposed Nodes if standard method is applied. As you see in Fig.4, in case of standard method with $5 \times 5$ grid, gray nodes (i.e. Exposed Nodes) are very often located at the boundary of the network. It is anticipated that boundary condition would affect throughput improvement ratio especially to small size grid. Therefore we have simulated up to $15 \times 15$ grid of 255 nodes.

\section{B. Simulation Result}

As shown in Fig.5, throughput per node goes lower as the size of grid goes bigger for both standard and proposed method. The simulation result showed that the proposed method achieved higher throughput per node in all grid sizes.

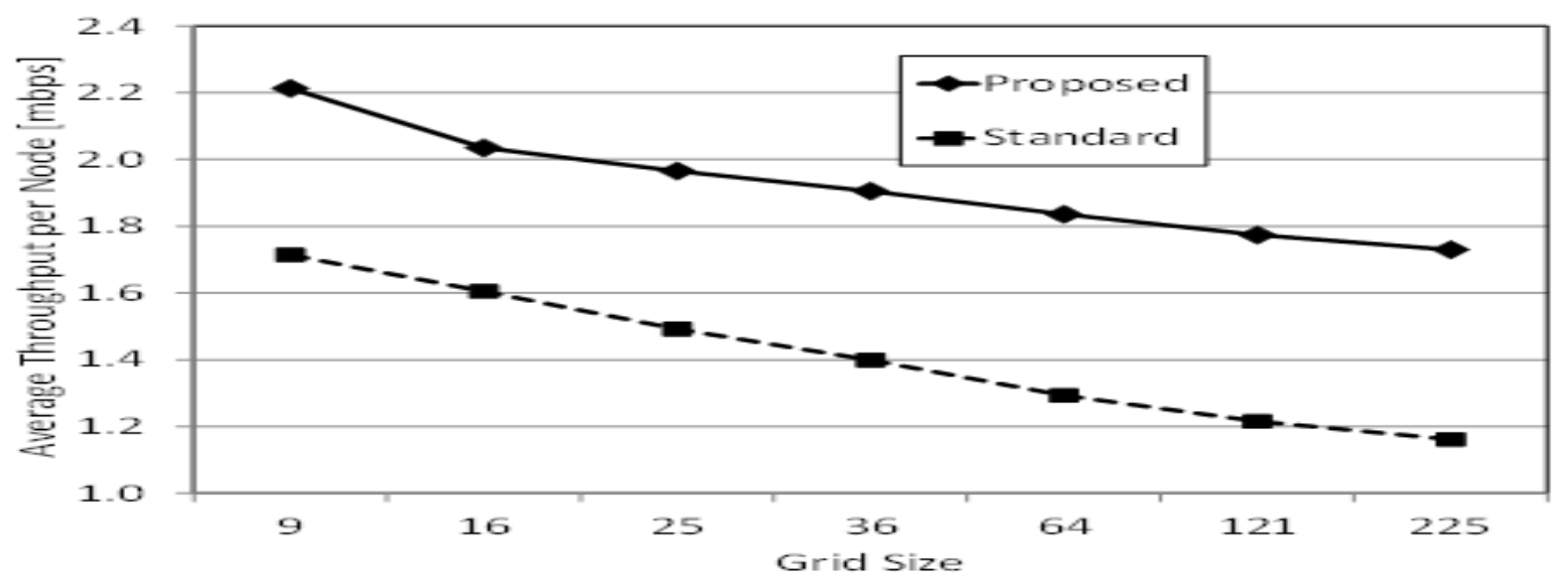

Fig.5 Average Throughput per Node 


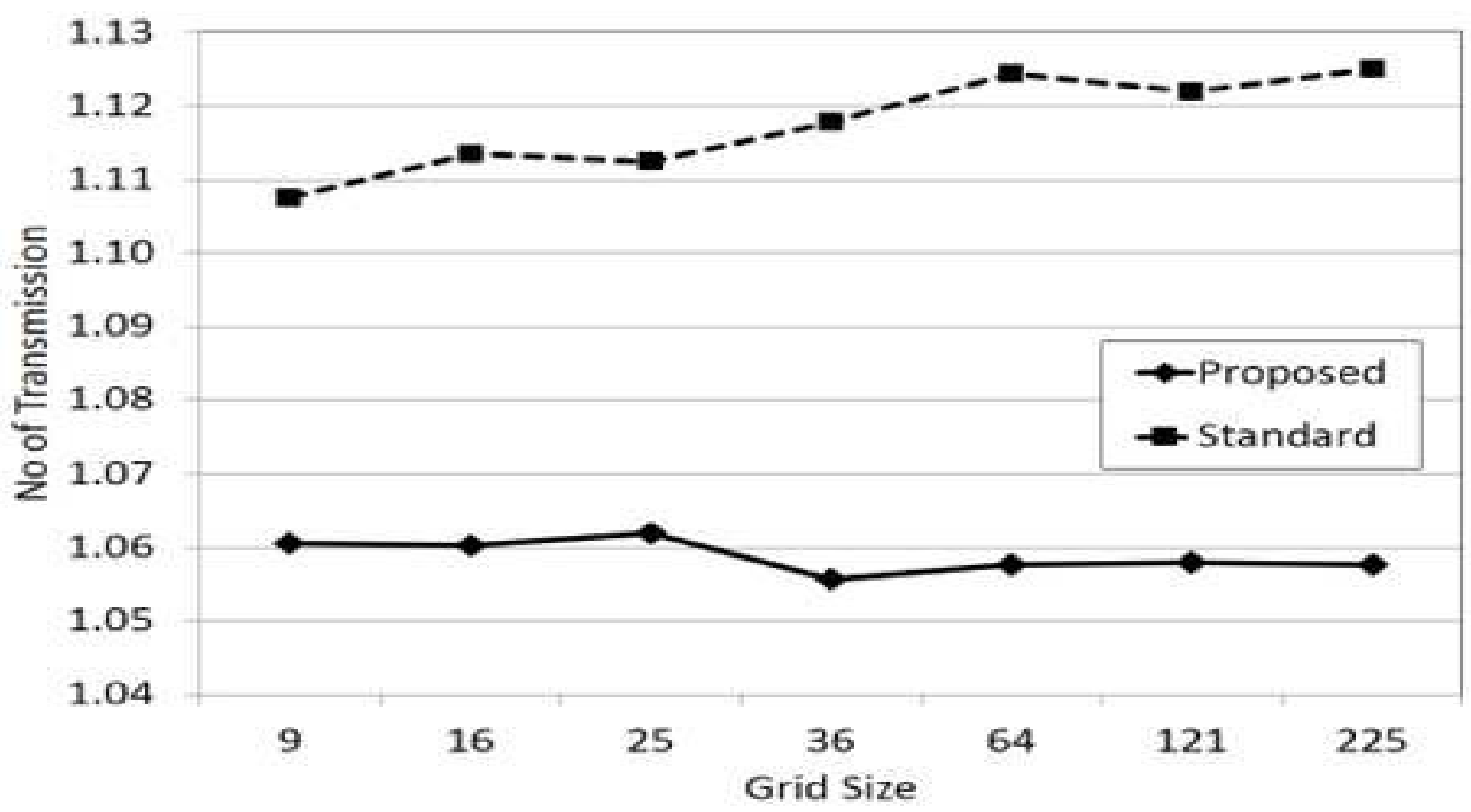

Fig.6 Average Number of RTS Transmission

Fig.6 shows average number of RTS transmission per data frame for each grid size. The number greater than 1.0 imply the occurrence of RTS retransmission. With standard method, $11 \%$ to $13 \%$ of RTS were retransmitted due to collisions. With the proposed method, only $5 \%$ to $6 \%$ of RTS were retransmitted.

Fig. 7 shows the average throughput dispersion. The proposed method has smaller dispersion than standard method, and this tendency is more ostensible with smaller grid size.

\section{CONClusion}

In this paper we have proposed the new method to adjust the transmitting rate of RTS to the same as data frame in order to control its transmission range proactively.

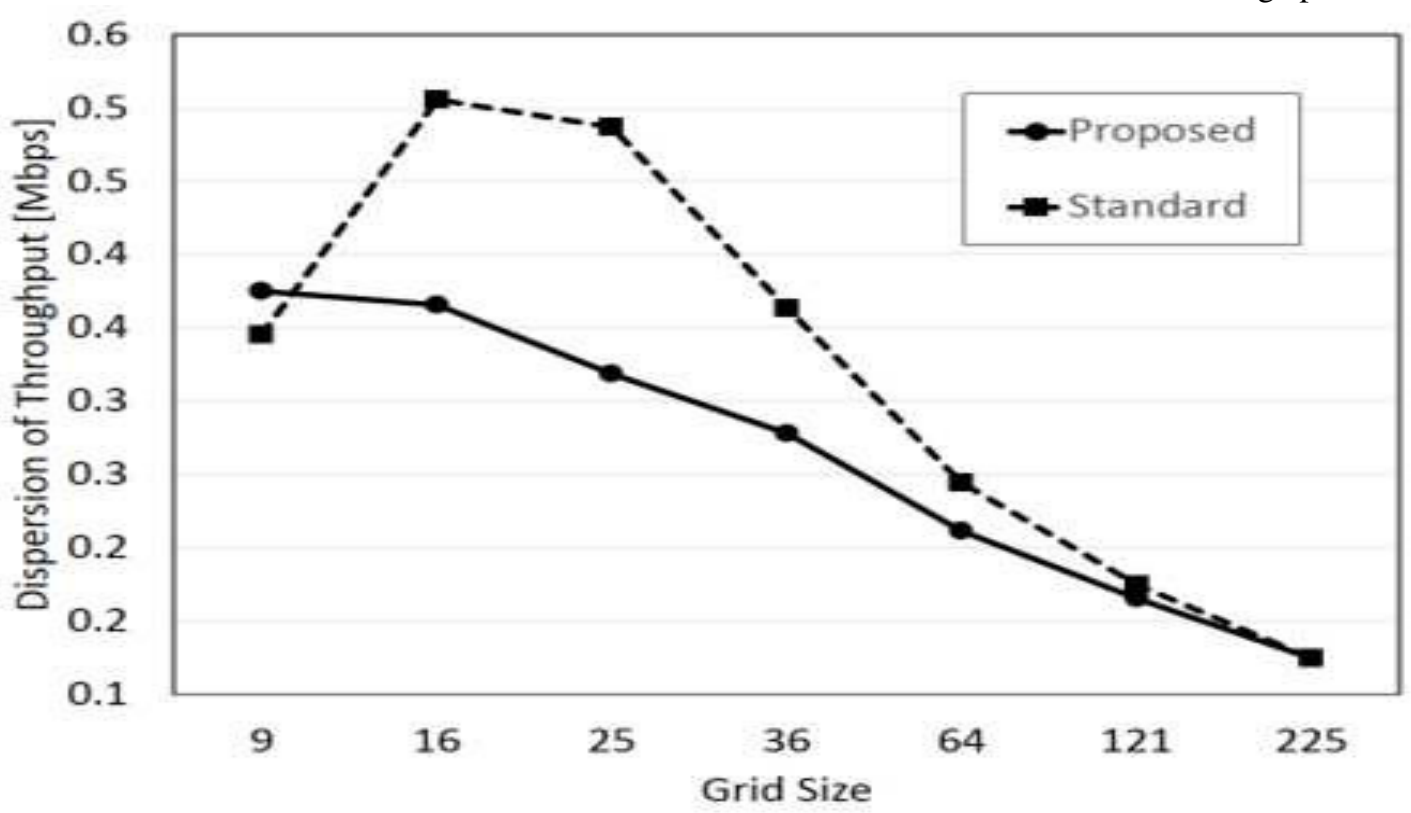

Fig.7 Dispersion of Throughput 
We have showed by simulation that the proposed method can improve throughput. We need to investigate further to validate effect of proposed method and to find method of selecting appropriate parameters as well as theoretical explanation.

\section{REFERENCES}

[1] K. Xu, M. Gerla, and S. Bae, "How effective is the IEEE802.11 RTS/CTS Handshake in Ad Hoc Network?" Proc. IEEE Globe Com’02, pp.72-76,Nov. 2002.
[2] S. Ray, J. B. Carruthers. and D. Starobinski, "RTS/CTS-Induced Congestion in Ad Hoc Wireless LANs," WCNC 2003 IEEE, vol. 3, pp.1516-1521, March 2003

[3] IEEE-SA Standards board, "Part11: Wireless LAN Medium Access Control (MAC) and Physical Layer (PHY) Specifications," IEEE Std 802.11-2012

[4] K. Nishide, H. Kubo, and R. Shinkuma, "Detecting Hidden and Exposed Terminal Problems in Densely Deployed Wireless Networks," IEEE Transactions on Wireless Communications, vol. 11, no. 11, pp.3841-3849, November 2011 